\title{
Synthesis of 3,4-disaccharides from pyranosides and furanosides monomers, a novel class of potential bioactive disaccharides
}

\author{
Muhammad Abbas ${ }^{\mathrm{a}}$, Bernhard Westermann ${ }^{\mathrm{a}, *}$, and Wolfgang Voelter ${ }^{\mathrm{b}, *}$ \\ ${ }^{a}$ Leibniz-Institute of Plant Biochemistry, Bioorganic Chemistry, Weinberg 3, 06120 Halle/Saale, \\ Germany \\ ${ }^{b}$ Interfakultäres Institut für Biochemie, Eberhard-Karls-University of Tübingen, \\ 72076 Tübingen, Germany \\ E-mail: bwesterm@ipb-halle.de
}

\section{Dedicated to Professor Attar-ur-Rahman on the occasion of his $65^{\text {th }}$ birthday}

\begin{abstract}
A new class of synthetically and pharmacologically important disaccharides containing epoxide moieties were synthesized from pyranoside and furanoside monomers in good to excellent yields. The scope and limitations for the formation of the linkage were evaluated.
\end{abstract}

Keywords: Carbohydrates, carbohydrate mimics, bioactive, disaccharides

\section{Introduction}

The defined topology and stereochemistry of carbohydrate frameworks permit an appealing array to synthesize natural products or natural-like products with interesting biological and pharmaceutical properties. ${ }^{1,2}$ Suitable intermediates and building blocks should bear appropriate functional groups for further transformations and in this respect, the oxirane is an ideal functional residue for subsequent regio- and stereoselective manipulations. ${ }^{3}$ Amongst others, conversions of the oxirane to trithiocarbonates, thiazolidenes, thiazolines, deoxy sugars, deoxy azido sugars and glycopeptides have been carried out successfully. ${ }^{4-9}$ Apart from the synthetic importance epoxide modified carbohydrates have been shown to exhibit several biological activities. ${ }^{10-16}$ This has been demonstrated on 2,3-epoxypropyl- $\beta$-D-glucosides and its oligomers which are inhibitors of hen's eggs lysozyme. ${ }^{10,11}$ Its epimer, the 2,3-epoxypropyl- $\alpha$-Dglucopyranoside irreversibly inhibits yeast hexokinase. ${ }^{12}$ Similarly, derivatives of cellobiose and cellotriose show inhibiting activity against cellulase. ${ }^{13}$ Furthermore, 3-O-alkyl 1,2-Oisopropylidene- $\alpha$-D-glucofuranoside can reduce intra-membrane charge movement, required for signal transduction between the sarcolemma and sarcoplastic reticulum, thereby inhibiting the 
excitation-calcium release mechanism (muscle relaxant activity). ${ }^{14}$ Oxirane ring-containing carbohydrates and a variety of sugar derivatives have been examined as possible inhibitors for a new thermostable neutral proteinase, isolated from Saccharomonospora canesencs. ${ }^{15}$ Among all tested compounds, benzyl 2,3-anhydro- $\beta$-L-ribopyranoside and benzyl 2,3-anhydro- $\alpha$-Dribopyranoside (11) exhibited the highest inhibiting activity.

\section{Results and Discussion}

Table 1. Synthesis of 3,4-disaccharides

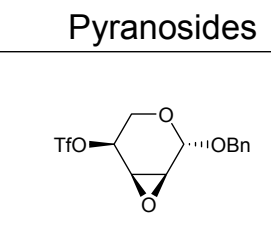

1

TfO"<smiles>[R]1CC2COC(O1)O2</smiles>

1

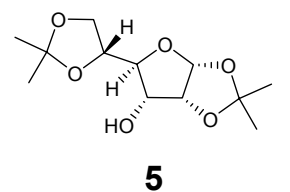

5

2

5

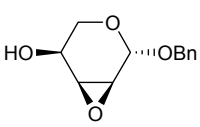

3

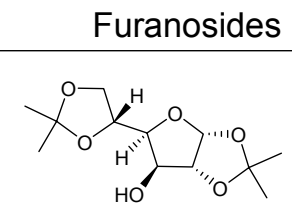

4

4

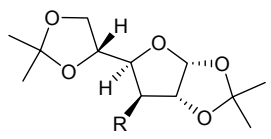

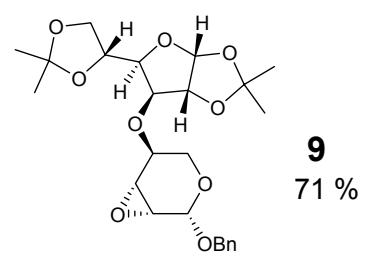
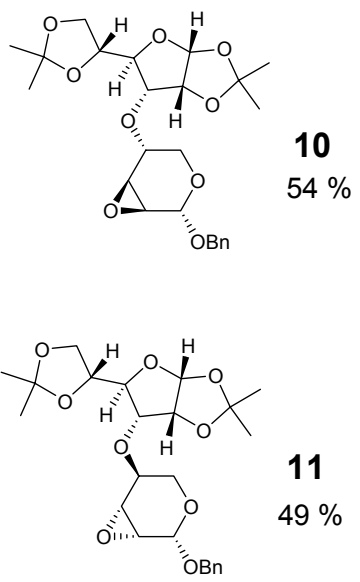

8

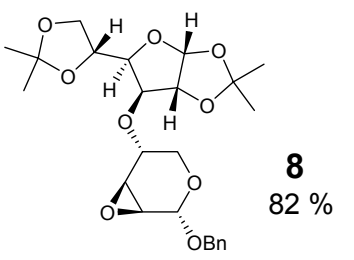

Disaccharides

$1 \%$

$\%$

no disaccharide

$6 \mathrm{R}=\mathrm{OTs}$

$7 \mathrm{R}=\mathrm{OTf}$

Reaction conditions: $\mathrm{Na}_{2} \mathrm{CO}_{3}, \mathrm{CH}_{3} \mathrm{CN}, 0{ }^{\circ} \mathrm{C} \rightarrow 20^{\circ} \mathrm{C}$, 7 h. 
This paper describes the successful coupling of pyranosides ( $\beta$-L-ribopyranoside 1 and $\alpha$-Dribopyranoside 2) with furanosides ( $\alpha$-D-furanoside 4 and $\alpha$-D-allofuranoside 5 ). The epoxy sugars were prepared according to literature procedures, starting from L-arabinose and Darabinose, respectively. ${ }^{16,17}$ The furanosides $\mathbf{4}$ and $\mathbf{5}$ were prepared as according to literature procedures. $^{18}$

As reported earlier by our group, a variety of sugar derivatives exhibit thermostable neutral proteinase inhibition. ${ }^{3}$ Considering this, we have decided to couple the glucopyranosides $\mathbf{4}, \mathbf{5}$ and 6 with the epoxytriflates 1 and 2, respectively, to obtain new disaccharides as shown in Table 1. The disaccharides 8 - 11 were synthesized by nucleophilic attack of $\mathbf{4}$ and $\mathbf{5}$, respectively, using mild coupling conditions $\left(\mathrm{Na}_{2} \mathrm{CO}_{3} / \mathrm{CH}_{3} \mathrm{CN}\right)$ at $\mathrm{C}-4$ of the epoxytriflates 1 and 2 . The yields of these coupling reactions were in the range of $50-80 \%$. Employing pyranoside 3 in a reverse reaction setup, no disaccharide formation could be observed via nucleophilic substitution of the tosyl residue in $6{ }^{19}$ nor triflate residue in $7 .^{20}$. It can be assumed that steric hindrance at C-3 of the furanoside $\mathbf{6}$ and $\mathbf{7}$ is responsible for the unsuccessful reaction.

We have also carried out partial deprotection of $\mathbf{8}$ leading to glycerol derivative 12. These deprotected carbohydrate building blocks may find further applications as bidentate ligands (Scheme 1).
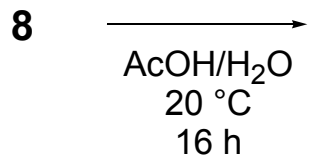

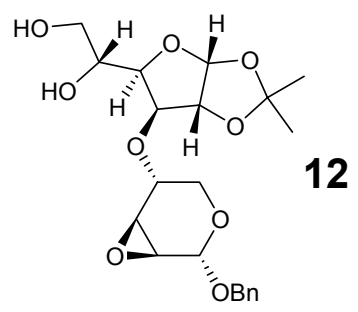

Scheme 1. Synthesis of partially deprotected derivatives.

After deprotection, which can be done under classical conditions, the dissaccharides will be further evaluated in biological tests.

\section{Experimental Section}

General Procedures. All air and moisture-sensitive reactions were performed under argon using dried glassware. Solvents were dried over standard drying agents. TLC (silica gel 60), precoated aluminium plates (F254) and silica gel 60 (40-630 mesh ASTM) were purchased from Merck, Germany. Mass spectra were measured on JMSHX-110 (Jeol), NMR spectra were obtained using Bruker AMX-250 MHz. 


\section{General Method for the synthesis of 3,4-disaccharides}

To a suspension of $1.2 \mathrm{mmol}$ of $\mathrm{Na}_{2} \mathrm{CO}_{3}(127 \mathrm{mg})$ in $5 \mathrm{~mL}$ dry $\mathrm{CH}_{3} \mathrm{CN}$ was added $1.2 \mathrm{mmol}$ of 4 $(312 \mathrm{mg})$ at $0^{\circ} \mathrm{C}$. The mixture was stirred for 30 minutes and then $1.0 \mathrm{mmol}(354 \mathrm{mg})$ of epoxy triflate dissolved in $2 \mathrm{ml}$ of $\mathrm{CH}_{3} \mathrm{CN}$, was added dropwise into the reaction mixture at $0^{\circ} \mathrm{C}$ which was stirred at this temperature for 30 minutes and then at room temperature for $6.5 \mathrm{~h}$. After completion of the reaction, as indicated by TLC, the reaction mixture was neutralized with a saturated solution of $\mathrm{NH}_{4} \mathrm{Cl}(50 \mathrm{~mL})$ and extracted with ethyl acetate $(2 \mathrm{X} 100 \mathrm{~mL})$, dried over anhydrous $\mathrm{Na}_{2} \mathrm{SO}_{4}$, filtered, concentrated to a syrup, and finally purified by column.

Benzyl 2,3-anhydro-4-O-(1,2:5,6-di-O-isopropylidene-3-deoxy- $\alpha$-D-glucofuranos-3-yl)- $\alpha$-Dlyxopyranoside (8). Purified by column chromatography in $82 \%$ yield using ethyl acetate:dichloromethane $(1: 9, \mathrm{v} / \mathrm{v})$. Colourless oil; ${ }^{1} \mathrm{H}$-NMR $\left(250 \mathrm{MHz}, \mathrm{CDCl}_{3}\right): \delta=7.35-7.20$ (5H-aromatic), $5.82(\mathrm{~d}, J=3.6 \mathrm{~Hz}, 1 \mathrm{H}), 4.92(\mathrm{~s}, 1 \mathrm{H}), 4.73\left(\mathrm{~d}, J=11.6 \mathrm{~Hz}, 1 \mathrm{H},-\mathrm{CH}_{2} \mathrm{Ph}\right), 4.51$ $(1 \mathrm{H}, \mathrm{d}=J 3.3 \mathrm{~Hz}, 1 \mathrm{H}), 4.48\left(\mathrm{~d}, J=11.6 \mathrm{~Hz} 1 \mathrm{H},-\mathrm{CH}_{2} \mathrm{Ph}\right), 4.16-3.21(\mathrm{~m}, 10 \mathrm{H}), 3.25(\mathrm{~d}, J=3.6$ $\mathrm{Hz}, 1 \mathrm{H}), 3.07$ (d, $J=3.7 \mathrm{~Hz}, 1 \mathrm{H}) .13 \mathrm{C} \mathrm{NMR}\left(62.8 \mathrm{MHz}, \mathrm{CDCl}_{3}\right) \delta$ : $105.3\left(\mathrm{C}-1^{\prime}\right), 93.8(\mathrm{C}-1)$,

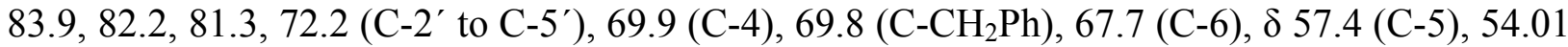
(C-3), 49.9 (C-2), 26.8, 26.8, 26.2, $25.3\left(4 \mathrm{xCH}_{3}\right)$. FAB-MS: e/z $465.3\left(\mathrm{M}^{+}\right), \mathrm{C}_{24} \mathrm{H}_{32} \mathrm{O}_{9},[\alpha]^{\mathrm{D}}=$ $+36.3^{\circ}\left(\mathrm{c}=1, \mathrm{CH}_{2} \mathrm{Cl}_{2}\right)$. Calculated C 62.06, H 5.94\%, Found C 62.49, H 6.02\%

Benzyl 2,3-anhydro-4-O-(1,2:5,6-di- $O$-isopropylidene-3-deoxy- $\alpha$-D-glucofuranos-3-yl)- $\beta$-L lyxopyranoside (9). Purified by column chromatography in $71 \%$ yield using ethyl acetate:dichloromethane (5:95, v/v). Colourless oil; ${ }^{1} \mathrm{H}$-NMR $\left(250 \mathrm{MHz}, \mathrm{CDCl}_{3}\right) \delta$ : 7.40-7.26 (5H-aromatic), 5.90 (d, J 3.6 Hz, 1H, H-1'), 5.06 (d, J $3.1 \mathrm{~Hz}, 1 \mathrm{H}, \mathrm{H}-1), 4.80$ (d, J 12.2 Hz, 1H, $\mathrm{CH}_{2} \mathrm{Ph}$ ), 4.62 (d, J $\left.12.2 \mathrm{~Hz}, 1 \mathrm{H}, \mathrm{CH}_{2} \mathrm{Ph}\right), 4.65$ (d, J $\left.3.7 \mathrm{~Hz}, 1 \mathrm{H}\right), 4.62$ - 4.34 (m, 1H), 4.15 - 3.86 (m, 6H), 3.35 (br. d, J $12.2 \mathrm{~Hz}, 1 \mathrm{H}), 3.40$ (m, 1H), $1.49\left(\mathrm{~s}, \mathrm{CH}_{3}\right), 1.41\left(\mathrm{~s}, \mathrm{CH}_{3}\right), 1.33\left(\mathrm{~s}, \mathrm{CH}_{3}\right)$, $1.31\left(\mathrm{~s}, \mathrm{CH}_{3}\right) .{ }^{13} \mathrm{C} \mathrm{NMR}\left(62.8 \mathrm{MHz}, \mathrm{CDCl}_{3}\right) \delta$ : 127.8-128.4 (5xC-Aromatic), 105.5 (C-1'), 92.8 (C-1), 84.0,82.2, 81.3, 72.5 (C-2' to C-5'), 72.4 (C-4), 69.3 (C-6'), $67.8\left(\mathrm{CH}_{2} \mathrm{Ph}\right), 59.1(\mathrm{C}-5)$, 51.6 (C-3), 50.1 (C-2), 26.9, 26.8, 26.3, $25.4\left(4 \mathrm{xCH}_{3}\right)$. FAB-MS: e/z $465.3\left(\mathrm{M}^{+}\right) \mathrm{C}_{24} \mathrm{H}_{32} \mathrm{O}_{9}$. Calculated C 62.06, H 5.94\%, Found C 62.09, H 6.08\%

Benzyl 2,3-anhydro-4-O-(1,2:5,6-di- $O$-isopropylidene-3-deoxy- $\alpha$-D-allofuranos-3-yl)- $\alpha$-Dlyxopyranoside (10). Purified by column chromatography in 54\% yield using ethylacetate:dichloromethane (1:9, v/v). ${ }^{1} \mathrm{H}$-NMR $\left(250 \mathrm{MHz}, \mathrm{CDCl}_{3}\right) \delta: 7.35$ - $7.20(5 \mathrm{H}-$ aromatic), 5.73 (d, J $3.6 \mathrm{~Hz}, 1 \mathrm{H}, \mathrm{H}-1^{\prime}$ ), 5.22 (br.-s, 1H), 4.92 (br.-s, 1H), 4.77 (br. d, J $11.9 \mathrm{~Hz}$, $1 \mathrm{H}), 4.56-4.47(\mathrm{~m}, 2 \mathrm{H}), 4.33-4.26(\mathrm{~m}, 1 \mathrm{H}), 4.05-3.92(\mathrm{~m}, 4 \mathrm{H}), 3.76-3.70(\mathrm{~d}, J 6.1$ and $9.7 \mathrm{~Hz}$ $1 \mathrm{H}), 3.62-3.50(\mathrm{~m}, 2 \mathrm{H}), 3.37(\mathrm{~d}, J 3.6 \mathrm{~Hz} 1 \mathrm{H}), 3.05(\mathrm{~d}, J 3.3 \mathrm{~Hz}, 1 \mathrm{H}), 1.47\left(\mathrm{~s}, \mathrm{CH}_{3}\right), 1.40$ (s, $\left.\mathrm{CH}_{3}\right), 1.29\left(\mathrm{~s}, \mathrm{CH}_{3}\right), 1.28\left(\mathrm{~s}, \mathrm{CH}_{3}\right) ;{ }^{13} \mathrm{C} \mathrm{NMR}\left(62.8 \mathrm{MHz}, \mathrm{CDCl}_{3}\right) \delta: 104.1\left(\mathrm{C}-1^{\prime}\right), 93.9(\mathrm{C}-1)$, 78.5, 78.1, 78.0, 74.7 (C-2' to C- 5'), 69.2 (C-4), 69.8 (C-CH2Ph), 65.3 (C-6'), 57.9 (C-5), 53.8 (C-3), 49.9 (C-2), 26.8, 26.7, 26.3, $24.7\left(4 \mathrm{xCH}_{3}\right)$. , FAB-MS: e/z $465.3\left(\mathrm{M}^{+}\right) \mathrm{C}_{24} \mathrm{H}_{32} \mathrm{O}_{9},[\alpha]^{\mathrm{D}}=$ $+91.2^{\circ}\left(\mathrm{c}=1, \mathrm{CH}_{2} \mathrm{Cl}_{2}\right)$. Calculated C 62.06, H 5.94\%, Found C 62.19, H 6.90\%

Benzyl 2,3-anhydro-4-O-(1,2:5,6-di- $O$-isopropylidene-3-deoxy- $\alpha$-D-allofuranos-3-yl)- $\beta$-Llyxopyranoside (11). Purified by column chromatography in 49\% yield using ethyl acetate:dichloromethane (1:9, v/v). Colourless oil; ${ }^{1} H$-NMR $\left(250 \mathrm{MHz}, \mathrm{CDCl}_{3}\right) \delta$ : 7.32-7.19 
(5H-aromatic), 5.77 (d, J $\left.3.6 \mathrm{~Hz}, 1 \mathrm{H}, \mathrm{H}-1^{\prime}\right), 4.99$ (d, J 3.1, 1H, H-1), 4.77 (d, J $12.2 \mathrm{~Hz}, 1 \mathrm{H}$, $\left.\mathrm{CH}_{2} \mathrm{Ph}\right), 4.58$ (d, J $\left.12.2 \mathrm{~Hz}, 1 \mathrm{H}, \mathrm{CH}_{2} \mathrm{Ph}\right), 4.01-3.96$ (m, 3H), 3.85 (br. s, 2H), 3.52 (s, 3H), 3.29 $(\mathrm{m}, 1 \mathrm{H}), 1.49\left(\mathrm{~s}, \mathrm{CH}_{3}\right), 1.39\left(\mathrm{~s}, \mathrm{CH}_{3}\right), 1.31\left(\mathrm{~s}, \mathrm{CH}_{3}\right), 1.29\left(\mathrm{~s}, \mathrm{CH}_{3}\right) . \mathrm{FAB}-\mathrm{MS}: \mathrm{e} / \mathrm{z} 465.3\left(\mathrm{M}^{+}\right)$, $\mathrm{C}_{24} \mathrm{H}_{32} \mathrm{O}_{9},[\alpha]^{\mathrm{D}}=+86.1^{\circ}\left(\mathrm{c}=1, \mathrm{CH}_{2} \mathrm{Cl}_{2}\right)$. Calculated C 62.06, H 5.94\%, Found $\mathrm{C} 62.40, \mathrm{H} 6.00 \%$ Benzyl 2,3-anhydro-4-O-(1,2-O-isopropylidene-3-deoxy- $\alpha$-D-glucofuranos-3-yl)- $\alpha$-Dlyxopyranoside (12). Prepared by stirring overnight $0.1 \mathrm{mmol}$ of 8 (31.2 $\mathrm{mg}$ ) with $20 \%$ acetic acid $(2 \mathrm{~mL})$ at room temperature. Purification by column chromatography afforded 12 in $92 \%$ yield using ethyl acetate:dichloromethane (2:8, v/v). Colourless oil; ${ }^{1} \mathrm{H}$-NMR (250 MHz), $\mathrm{CDCl}_{3}$ ) 7.37-7.26 (5H-aromatic), 5.93 (d, $\left.J=3.6 \mathrm{~Hz}, 1 \mathrm{H}, \mathrm{H}-1^{\prime}\right), 4.97$ (d, $J=3.1 \mathrm{~Hz}, 1 \mathrm{H}, \mathrm{H}-1$ ), $4.85\left(\mathrm{~d}, J=12.2 \mathrm{~Hz}, 1 \mathrm{H}, \mathrm{CH}_{2} \mathrm{Ph}\right), 4.58\left(\mathrm{~d}, J=12.2 \mathrm{~Hz}, 1 \mathrm{H}, \mathrm{CH}_{2} \mathrm{Ph}\right), 4.18-4.12(\mathrm{~m}, 3 \mathrm{H}), 3.95-$ $3.80(\mathrm{~m}, 2 \mathrm{H}), 3.27-3.57(\mathrm{~m}, 3 \mathrm{H}), 3.32(\mathrm{~s}, 1 \mathrm{H}), 3.30(\mathrm{~s}, 1 \mathrm{H}), 3.16$ (brs, $1 \mathrm{H}), 1.49\left(\mathrm{~s}, \mathrm{CH}_{3}\right), 1.25$ $\left(\mathrm{s}, \mathrm{CH}_{3}\right) .{ }^{13} \mathrm{C} \mathrm{NMR}\left(62.8 \mathrm{MHz}, \mathrm{CDCl}_{3}\right)$ 128.5-128.1 (5 x C, aromatic), 105.1 (C-1'), $94.4(\mathrm{C}-1)$, 83.0, 81.2, 80.0, $70.4\left(\mathrm{C}-2^{\prime}\right)$ to $\left.\mathrm{C}-5^{\prime}\right), 69.2(\mathrm{C}-4), 68.8\left(\mathrm{C}-\mathrm{CH}_{2} \mathrm{Ph}\right), 64.5\left(\mathrm{C}-6^{\prime}\right), 57.2(\mathrm{C}-5), 52.8$ (C-3), 26.7, $26.3\left(2 \times \mathrm{CH}_{3}\right)$.

\section{Acknowledgements}

We would like to acknowledge DAAD (Deutscher Akademischer Austauschdienst) for providing funds for this project through a sandwitch $\mathrm{PhD}$ scholarship.

\section{References and Footnotes}

1. Ferrier, R. J., Middleton, S. Chem. Rev. 1993, 93, 2779 and references therein.

2. Abed, M. Y.; Naz, N.; Mootoo, D.; Voelter, W. Tetrahedron Lett. 1996, 37, 8641.

3. Abed-Jalil, R. J.; Read, A.; Al-Qawasmeh.; Voelter, W. Tetrahedron Lett. 1998, 39, 6155 and references therein.

4. Saeed, M.; Abbas, M.; Zahid, M.; Voelter, W. Tetrahedron Lett. 2003 , 44, 315.

5. Saeed, M.; Abbas, M.; Voelter, W. Tetrahedron Lett. 2003, 44, 6107

6. Abed-Jalil, R. J.; Saeed, M.; Voelter, W. Tetrahedron Lett. 2001, 42, 2435.

7. Heskell, T. H.; Woo, P. W.; Watson, D. R. J. Org. Chem. 1977, 42, 1302.

8. Kimmich, R.; Voelter, W. Liebigs Ann. Chem. 1981, 1100.

9. Hurthley, S.; Service, R.; Szuromi, P. Science 2001, 291, 2337.

10. Thomas, E. W. Carbohydr. Res. 1970, 13, 225.

11. Marone, Y.; Eshadat.; Sharon, N. Biochem. Biophys. Acta 1972, $278,243$.

12. Bessell, E. M.; Westwood, J. H. Carbohydr. Res. 1972, 25, 11.

13. Legier, G.; Baose, E. Carbohydr. Res. 1973, 28, 45.

14. Becaert, T.; Villa, P.; Boorn, Roland. Biomed. Res. 1994, 15, 1, $28,45$.

15. Huber, R. E.; Brockbank.; Strong, R. L. Biochemistry 1987, 26, 1526. 
16. Fletcher, H. G.; Jr.; Hudson, C. S. J. Am. Chem. Soc. 1950, 72, 4173.

17. Fletcher, H. G.; Jr.; Hudson, C. S. J. Am. Chem. Soc. 1947, 69, 1145.

18. Schmidt, O. T. Carbohydr. Chem. 1963, 2, 318.

19. Valverde, S.; Hernandez, A.; Herradon, B.; Rabanac, R. M.; Martin-Lomas, M. Tetrahedron 1987, 43, 3499.

20. Achab, S.; Das, B. C. J. Chem. Soc., Perkin Trans. 1 1990, 2863.

21. Maurus, S. Helv. Chem. Acta. 1993, 76, 1832. 